\title{
Germanica
}

Der Zeitroman in beiden Deutschland (1971-1991) auf dem Wege der kulturellen Wiederannäherung

Le roman d'actualité dans les deux Allemagnes (1971-1992)

\section{Volker Wehdeking}

\section{OpenEdition}

\section{Journals}

Édition électronique

URL : http://journals.openedition.org/germanica/2223

DOI : $10.4000 /$ germanica.2223

ISSN : 2107-0784

Éditeur

Université de Lille

Édition imprimée

Date de publication : 1 décembre 1994

Pagination : 149-175

ISSN : 0984-2632

Référence électronique

Volker Wehdeking, « Der Zeitroman in beiden Deutschland (1971-1991) auf dem Wege der kulturellen Wiederannäherung », Germanica [Online], 14 | 1994, Online erschienen am: 20 Januar 2014, abgerufen am 06 Oktober 2020. URL : http://journals.openedition.org/germanica/2223 ; DOI : https://doi.org/ 10.4000/germanica.2223

Ce document a été généré automatiquement le 6 octobre 2020.

(ㄷ) Tous droits réservés 


\title{
Der Zeitroman in beiden Deutschland (1971-1991) auf dem Wege der kulturellen Wiederannäherung
}

\author{
Le roman d'actualité dans les deux Allemagnes (1971-1992)
}

Volker Wehdeking

1 In den frühen Jahren nach der Gründung beider Deutschland (1949 bis zum vorübergehenden Tauwetter der ersten Honecker-Jahre nach dem 8. Parteikongreß 1971) gab es zunächst eine weitgehend unter ideologischen Vorzeichen in Ost und West verschiedene Kulturentwicklung. In der Bundesrepublik entwickelte sich eine spätmoderne, lange von der Gruppe 47 dominierte Literatur in einem Staat konservativ-liberalen Bürgertums. In der DDR wurden sich die durch einen rigorosen «Sozialismus von oben» gegängelten Künstler und Schriftsteller bereits nach dem Mauerbau ihrer massiv eingeschränkten Kreativität bewußt und fanden im verspäteten Anschluß an die Klassische Moderne und in der - teilweise subtil verdeckten Opposition zur herrschenden Ideologie zu einer kulturellen Wiederannäherung an die westliche Kulturentwicklung.

Diese «kodierte» DDR-Literatur versuchte auch ästhetisch, durch den Anschluß nicht nur an die internationale Spätmoderne, sondern an eine gemeinsame ältere deutsche Literaturtradition, die im sozialistischen Realismus kaum Gegenliebe fand, Opposition zur staatstragenden Literaturszene zu signalisieren: gemeint ist die Tradition (nach der germanistischen Behelfsperio-disierung) «zwischen Klassik und Romantik», der darin zusätzlich betonten, subjektivistischen und frauenspezifischen Komponente und des forminnovativen Vormärz der Grabbe und Büchner - ich nenne hier stellvertretend Christa Wolf, Volker Braun und Günter de Bruyn und deren Wiederaufnahme von Kleist, Jean Paul, Heine, und Büchner, von Karoline von Günderrode und Bettina von Arnim, sowie für die spätmodernen Montage-und Collageverfahren die Erzählprosa Reiner Kunzes, Monika Marons, Christoph Heins, die Dramen und Gedichte Heiner 
Müllers und die Lyrik von Günter Kunert und Sarah Kirsch. Schließlich wird in der kodierten DDR-Literatur ein Point-of-view nicht des «typischen» mittleren Helden präsentiert, sondern - etwa in der Pikaro-Tradition bei Irmtraud Morgner, Fritz Rudolf Fries oder Helga Königsdorf - die Außenseiterperspektive: jene verkrachter Bohémiens und wiedergeborener Troubadours, verhinderter Jazzliebhaber und in ihrer Berufsausübung behinderter Germanisten, kaltgestellter und überwachter Autoren und Journalisten, im Protest verstummter oder aus der Hochschule gedrängter Historiker und Historikerinnen auf imaginären oder phantastischen Fluchtreisen. Daß sich hier die Historiker-Protagonisten in den letzten Jahren vor dem Mauerfall in DDR-Romanen häufen, erscheint alles andere als zufällig. Ich komme darauf zurück. Vielleicht am eindrucksvollsten begleitet Wolfgang Hilbig in der Erzählprosa seit 1989 (Die Übertragung, Grünes, grünes Grab, 1992, Ich, 1993) mit an Kafka erinnernder Intensität die schizophrene Existenz von bespitzelten Schriftstellerprotagonisten, die sich mehr und mehr mit fingierten Vorwürfen identifizieren lernen und ihre Heimat immer weniger begreifen und wiedererkennen, ihr Heil aber dann doch im Westen suchen.

Bereits Mitte der Sechziger Jahre, nach Mauerbau und harter, auch kultureller Repression, begann sich die DDR-Literatur, zunächst subversiv, wieder auf die in diesen Jahren der 1968er Bewegung politisierte, teilweise operativ und systemkritisch gemeinte Literatur der Bundesrepublik zuzubewegen. Die seit 1976 mit der BiermannAusbürgerung zunehmende Welle von in den Westen übersiedelnden DDR-Autoren und Theaterleuten gaben der in «Neuer Subjektivität» befangenen, höchstens noch in der frauenspezifischen Thematik zeitkritischen, bundesdeutschen Literatur neue Impulse. Zugleich war die literarische Systemkritik innerhalb der DDR durch meist im Westen publizierte Texte und Stücke von Volker Braun, Christoph Hein, ansatzweise Heiner Müller und Monika Maron (vor ihrer Übersiedlung 1988) sowie einigen Prenzlauer Berg Autor(inn)en bis zum 9. November 1989 immer weniger zu überhören. Die nunmehr als Träger beider deutscher Literaturen verbleibende bürgerliche Schicht (in der späten Weimarer Republik noch den Radikalisierungen von links und rechts erlegen) tritt ein lebendiges kulturelles Erbe an und muß sich der Herausforderung stellen, die gegenseitige Wiederannäherung nicht durch einen Rückfall ins Gemütliche zu verspielen. Die Gefahr einer westlichen Erstarrung der Verhältnisse, wie sie Botho Strauß und Thomas Bernhard in den Achtziger Jahren zum Thema machten, dürfte aufgrund der Aporien «deutscher Befindlichkeiten» im Einigungsprozeß fürs erste gebannt sein.

Bei einem so weitgespanntem Thema ist Einschränkung geboten: mehr als zwanzig Jahre deutsch-deutscher Literatur, und dann auch noch ein kritischer Vergleich, das ist im Rahmen eines Vortrags nur in Abbreviaturen $\mathrm{zu}$ bewältigen, und selbst im kursorischen Überblick nur, wenn man die Kenntnis westdeutscher Literaturentwicklungen weitgehend voraussetzt. Eine Beschränkung innerhalb der Gattungen und im Thema ist ebenfalls unumgänglich: neben einigen wenigen Gedichten geht es um deutsche Erzählprosa vor allem aus der DDR und im Spannungsfeld der deutschen Teilung: denn immer schon gab es das Thema des Leidens von DDR-Künstlern und -Autoren an ideologischer Bevormundung, auch wenn sie den Sozialismus für eine lohnende Utopie und das System für ausbaufähig hielten. So geraten Handlungsabläufe von Romanen und Erzählungen in den Brennpunkt, in denen die Hauptfiguren sich nach kultureller Veränderung sehnen, nach Fortgang in den Westen, nach mehr kritischer Einmischung vor Ort, im Grunde immer wieder das Menschenrecht auf Meinungsfreiheit und Freizügigkeit reklamierend. Heute, vier Jahre 
nach der Selbstbefreiung der DDR am 9. November 1989 gibt es eine Reihe von gegenseitigen sozialen und politischen Vorbehalten der Neuen und Alten Bundesländer, die zeigen, daß eine kulturelle Wiederannäherung nach 40 Jahren Teilung alles andere als einfach ist. Vieles an der Aufbruchseuphorie Ende 1989 erscheint nun mit guten Gründen der Alltagsmisere eine noch viele Jahre andauernden Ungleichzeitigkeit gewichen. Für die sozio-politischen gegenseitigen Enttäuschungen hat der Kandidat der Grünen fürs Präsidentenamt, Jens Reich, in jüngster Zeit eine Reihe von plausiblen Gründen genannt. Er sieht die Krise der inneren Einheit in der Plötzlichkeit des Verlustes eigener Identität der Neuen Bundesländer auf fast allen Gebieten und, natürlich, im drohenden Verlust des Arbeitsplatzes:

Alles Gewohnte der täglichen Lebensgestaltung wurde schwankend, als das gesamte zivile Recht, Schuldrecht, Familienrecht, Erbrecht, Mietrecht, Eigentumsund Vermögensordnung, die soziale Verfassung (Ausbildung, Erziehung, Berufsordnung, Gesundheitsfürsorge, Rentenrecht und Alters vor sorge) schlagartig ausgewechselt wurde, wobei die Menschen weder die neue Ordnung kannten noch auch nur ihre spezifische Verwaltungssprache verstanden, also zu sekundären Analphabeten in ihrem Gemeinwesen wurden ${ }^{1}$.

5 Für den Literaturbetrieb denke man nur an die Schlagzeilen rund um die neue Berliner Akademie der Künste und die anhaltenden Austritte von Autoren und Künstlern aus beiden Teilen Deutschlands wegen der von Walter Jens und Heiner Müller nach langem Gezerre beschlossenen En-Bloc-Zuwahl der Ostberliner Akademiemitglieder von einst. Die im Januar 1993 bekannt gewordenen Stasi-Kontakte von Heiner Müller und Christa Wolf geben zusätzlich zu denken. Die berechtigten Einwände von Wolfgang Biermann gegen die schillernde Rolle eines für den Staatssicherheit spitzelnden Sascha Anderson (geb. 1953) oder Reiner Schedlinski (geb. 1956) - beide der angeblich alternativen Literaturszene des Prenzlauer Berg zugehörig- und deren postmoderne Sprachexperimente bringt Ralf Schnell in seiner neuen Geschichte der deutschsprachigen Literatur seit 1945 (1993) auf eine dennoch kulturell konvergierende Ebene:

Statt Verlautbarungen, Inhalten und Konventionen wurden nun [bei den Lyrikern vom Prenzlauer Berg] Strukturen, Konturen und Formationen in den Blick gerückt [...]: Die Hohlformen der östlichen Weltanschauungsdiktatur und die traditionsreichen Sinnentwürfe der westlichen Industriegesellschaften erwiesen sich unterderhand als strukturhomolog. [...].

Ein Teil der DDR-Literatur, so scheint es, wird von nun an auf einer zweifachen Ebene zu lesen sein - auf der ihrer ästhetischen Struktur, deren Polyvalenz auch traditioneller Hermeneutik zugänglich ist, und auf der von Verschlüsselungen und Ver rät seiungen, Codierungen und Geheimbotschaften, die von einer gespaltenen Künstlerexistenz künden. Gottfried Benns Begriff «Doppelleben» dürfte einer Untersuchung den passenden Titel bieten, die an solchen Texten das Problem der poetischen Wahrheit ausloten wollte ${ }^{2}$.

Wenn Wolfgang Emmerich in der letzten Auflage einer weit verbreiteten, einbändigen deutschen Literaturgeschichte ${ }^{3}$ von einem selbständigen westdeutschen literarischen Feld in den Jahren 1949-1989 mit einem «angeschlossenen Feld DDR-Literatur» spricht, stellt er die Homogenität des Feldes «Literatur der Bundesrepublik» zugleich in Frage, sieht sie seit längerem «ausgespannt zwischen den Polen 'Gewissen der Nation' und 'postmoderner Boulevard'» als «längst uneinheitlich geworden» und betont die Ähnlichkeit beider deutscher Literaturen in ihrer konvergierenden Thematik, bedingt durch ähnlicher werdende Probleme seit 1980 bei aller Ungleichzeitigkeit im einzelnen. Seit etwa 1980 kann man die beiden deutschen Literaturen in West und Ost auf einem Kurs der Wiederannäherung beobachten, der sich nicht nur in den vielen 
Ausbürgerungen und Ausreisen von DDR-Autoren und Autorinnen im Gefolge der Biermann-Solidarisierung niederschlägt. Man kann nun wieder mit Bour-dieu von einem literarischen Feld sprechen, da beide Literaturen nicht mehr ohne einander und nur in gegenseitiger Wahrnehmung gedacht werden können.

7 Am nachhaltigsten haben mich in diesem Zusammenhang die kulturellen Thesen und Überlegungen Günter de Bruyns in seinem Essayband Jubelschreie, Trauergesänge. Deutsche Befindlichkeiten (1991) beeindruckt. De Bruyn hält am Gedanken einer «deutschen Kulturnation» fest, weil für ihn die Kulturnation nicht beim Nationalstaat und Bismarck-Reich beginnt, sondern «vielleicht bis zu Luther» zurückreicht, «ganz deutlich aber in die Zeiten der Aufklärung»:

Als Gottsched in Leipzig seine «Deutsche Gesellschaft» gründete, Lessing sich in Hamburg um ein Theater bemühte, das der ganzen Nation gehören sollte, [...] und Herder erkannte, daß die Kulturen im weitesten Sinne (also die Sprachen, die Lieder die Dichtungen, Sitten, Gebräuche) es sind, die die Nationen bilden - war es schon da, dieses nicht unverwüstliche, aber doch langlebige Band, das in klassischer Zeit, die eine Zeit politischer Zerrissenheit war, ein nationales Zusammengehörigkeitsgefühl schuf. [...].

Von der Existenz einer deutschen Kulturnation auszugehen, zu der man gehört, ob man will oder nicht, scheint mir ehrlicher und objektiver als das Reden von nationalen Gefühlen, die die Massen angeblich bewegen oder auch angeblich nicht. [...] Der Begriff der Kulturnation [...], sozusagen metapolitisch [...] sagt aus, daß die Deutschen, durch Kultur und Geschichte bedingt, zusammengehören, aber über Grenzen, Verfassungsgrundsätze und Souveränitätsrechte sagt er nichts ${ }^{4}$.

8 Eine zweite These Günter de Bruyns berichtigt das Klischee des Kalten Krieges, die Westdeutschen seien amerikanisiert, die Ostdeutschen dementsprechend «russifiziert» worden:

Um über die Welt, in die man nicht reisen konnte, und auch über die DDR, in der man lebte, wahrheitsgemäß informiert zu werden, mußte man Programme westlicher Sender empfangen, lebte also auf diese Weise das Radio- und Fernsehleben des anderen Teilstaates mit. Der Preis, den man dafür zahlte, daß man tagsüber auf der Arbeitsstelle mehr oder weniger aufrichtig den Staats- und parteitreuen, also einheitsfeindlichen DDR-Bürger zeigte, am Abend sich aber mit den Deutschen jenseits der Grenze am Bildschirm vereinte, war eine kaum bewußte schizophrenie-ähnliche Persönlichkeitsspaltung, [...] Aus dem Mond fiel man also nicht, als im Herbst 1989 die Wende erfolgte; [...] Bei dem Versuch, ein Psychogramm der Bevölkerung der ehemaligen DDR zu geben, dürfen zwei weitere Tatsachen nicht fehlen, nämlich die Folgen der ideologischen Schulung und die durch Einmauerung erzeugte Provinzialität. [...] Man kennt die Welt nicht, auch nicht die östliche, obwohl man gelegentlich in sie reisen durfte und die Freundschaft mit den sozialistischen Ländern von der SED-Führung immer gefordert war. Vielleicht wegen dieses Verordnungscharakters der Freundschaft [...] gab es [...] nie [...] im Warschauer Pakt ein Zusammengehörigkeitsgefühl in den Bevölkerungen; die Regierungen waren verbündet, die Völker lebten voneinander abgeschottet. [...] Man blickte in der DDR über die Mauer nach Westen, aber das hieß in erster Linie nach Deutschland, wodurch zwar das Nationale gefördert wurde, aber nicht die Weltoffenheit ${ }^{5}$.

Der siebeneinhalb Jahre in der DDR aus politischen Gründen («konterrevolutionäre Gruppenbildung») inhaftierte, 1981 in den Westen gegangene Erich Loest dokumentiert die Zensur- und Überwachungsschikanen in zwei sprechenden Titeln: Der Zorn des Schafes. Aus meinem Tagewerk (Leipzig 1990) und Die Stasi war mein Eckermann oder: mein Leben mit der Wanze (Göttingen 1990). Der Hörspielpreis der Kriegsblinden ging 1991, damit eine ehrwürdige Nachkriegstradition verbindend, an das DDR-Hörspiel jenes 
Jahres, «Stille Helden singen selten», das Originaltöne der Montagsmärsche und Mauerkommentare mischt. Das Gefühl des Identitätsverlusts und der Orientierungsschwierigkeiten vieler DDR-Intellektuellen äußert sich aber ebenso deutlich. Von evokativer Kraft sind in dem Zusammenhang einige Gedichte von 1990 aus der Feder Heiner Müllers, des namhaftesten DDR-Dramatikers nach Brecht und Peter Hacks, und ein Atlantis-Gedicht Günter Kunerts aus dem Gedichtband mit dem sprechenden Titel Fremd daheim (1990). Müllers «Altes Gedicht» lautet, samt elegischem Motto, fast postmodern:

Ich bin der ich nicht sein will und der sein wird.

Nachts beim Schwimmen über den See der Augenblick

Der dich in Frage stellt

Es gibt keinen anderen mehr

Endlich die Wahrheit Daß du nur ein Zitat bist

Aus einem Buch, das du nicht geschrieben hast

Dagegen kannst du lange anschreiben auf dein

Verblassendes Farbband

Der Text schlägt durch.

Läßt sich hier ahnen, wie es um die schwindenden Themen und Identitäten der DDRAutoren und Autorinnen bestellt sein mag, so ist in dem wiederum sprechenden Titel Ein Gespenst verläßt Europa (Köln 1990) auch herbe «Selbstkritik» enthalten:
Meine Herausgeber wühlen in alten Texten
Manchmal wenn ich lese überläuft es mich kalt
Das habe ich geschrieben IM BESITZ DER WAHRHEIT
Sechzig Jahre vor meinem mutmaßlichen Tod
Auf dem Bildschirm sehe ich meine Landsleute
Mit Händen und Füßen abstimmen gegen die Wahrheit
Die vor vierzig Jahren mein Besitz war
Welches Grab schützt mich vor meiner Jugend.

Eine Erinnerung Müllers an die Hamletmaschine von 1977 und ein Plakat dazu lösen dieses nachdenklich machende Gedicht aus :

HAMLETMASCHINE: der Hamletdarsteller ohne Gesicht, im Rücken die Mauer, sein Gesicht eine Gefängniswand. Bilder, die keine Aufführung einholen konnte. Wegmarken durch den Sumpf, der sich schon damals zu schließen begann über dem vorläufigen Grab der Utopie, die vielleicht wieder aufscheinen wird, wenn das Phantom der Marktwirtschaft, die das Gespenst des Kommunismus ablöst, den neuen Kunden seine kalte Schulter zeigt, den Befreiten das/eiserne Gesicht seiner Freiheit.

Ebenso anrührend geht auf den Umbruch Günter Kunerts «Atlantis» ein, weil Kunert hier nicht nur in subtil indirekter Weise den Wechsel der Orts- und Straßenbenennungen seit 1989 anspricht, sondern als Sohn einer jüdischen Berliner Mutter auch den unvergessenen Holocaust, den die offizielle DDR-Kultur so lange als Problem dem Westen allein überließ. Das Gedicht von 1990 steht am Schluß des Bandes:

Atlantis

Als es unterging Sklaven

sollen geschrien haben in dieser Nacht

wie ihre Eigentümer: Homo sum.

Aber in den Legenden herrscht Stille

als wäre die Geschichte ein Konzert

gewesen und die Mitwirkenden heimgegangen.

Akten zur Beweisführung herbeigeschafft 
ihre blutige Schrift verblichen aber.

Erneut gelang die Transsubstantiation:

Alle Getöteten wurden zu annehmlichen Zahlen.

Heute schon sind für morgen die Fundstücke

frei von Konservierungsmitteln: Alles

Rätsel für Nachgeborene.

Wenn da wirklich geschrien wurde ist es ja

längst verhallt.

Amtlich als akustisches Phänomen eingestuft.

Als Präzedenzfall

für alle Zukunft wo wir geschrien haben werden.

Und die Unbetroffenen es deutlich überhört.

Und einige die Gelegenheit ergriffen

zu einem Gedicht: Spätfolge

von Untergängen die allein durch Ortsnamen

der Unterscheidung sich fügen ${ }^{6}$.

Hier hat Kunert das Gespenstische des Vorgangs in der Geschichte, die für alle unerwartet rasche öffnung der Mauer, ebenso festgehalten wie die untergegangene Heimat und längst abgewirtschaftete Utopie, an die sich der frühe Kunert klammerte ${ }^{7}$. Seine Elegie ist daher nachvollziehbar, obwohl er den Umbruch begrüßt. Hier gibt es natürlich ideologische Hintergründe, die bis in die frühe Nachkriegszeit zurückreichen.

\section{Das deutsch-deutsche Thema in ausgewählten Romanen, Erzählungen und Gedichten der DDR- Literatur seit 1971}

Hatten die beiden Bitterfelder Konferenzen 1959 und 1964 die Aktivistenporträts und das Ankommen im sozialistischen Alltag bis hin zum schreibenden Arbeiter für die Romanprosa gefordert, waren in dieser Gattung die intellektuellen Spannungen, die der Mauerbau 1961 und der sowjetische Einmarsch in Prag 1968 mit einem DDRSolidaritätsbeitrag auslöste, noch nicht so spürbar wie in der Lyrik seit 1965, etwa bei Günter Kunert oder Volker Braun.

In seinen Frankfurter Vorlesungen von 1985 blickt der seit 1979 in Holstein lebende Kunert auf die beginnende Enttäuschung und Erstarrung zurück, die bereits Mitte der 60er Jahre einsetzte und mit der Ausbürgerung Wolf gang Biermanns 1976 kulminierte:

Das Gedicht bot den kürzesten Weg. Und bewegt hat mich die zunehmende Unbeweglichkeit der Gesellschaft, die Erstarrung der sozialen Struktur. Was im ersten Jahrzehnt, vielleicht auch noch im zweiten nach Kriegsende wie eine tiefenwirksame Veränderung aussah, war wohl bloß eine Fluktuation zwischen sozialen Schichten. Es rückten in die Ämter und Posten, in die Verwaltung und in die Schaltstellen der Betriebe andere nach. Als ein bestimmter Sättigungsgrad erreicht war, versteinerte dieses Modell sofort, und diese gesellschaftliche Immobilität befiel natürlich irgendwann jede geistige Regsamkeit. [...] «Klassiker» ist ein Gedicht über Marx, von seinen Kindern «Mohr» genannt; eine Variation über das Thema «Marx heute»".

Klassiker

Rasiert und angestellt

sitzt im Büro

den Kopf in seiner Hand

entleert in lauter kleine Sprüche

die gigantischen Gedanken: Er. 


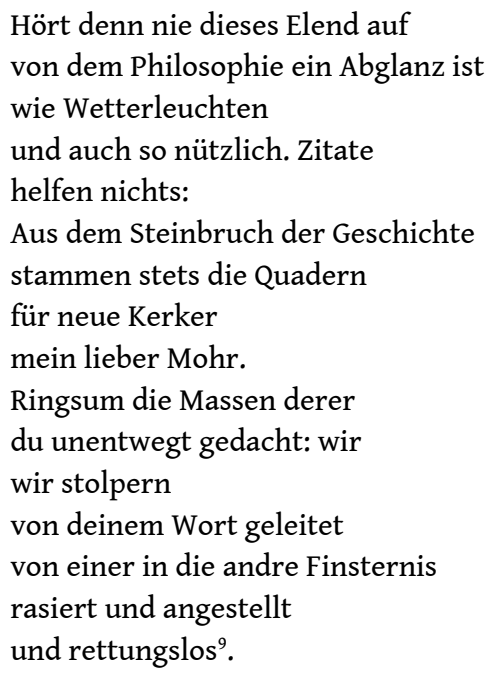

Ähnlich läßt Kunert dies Schwinden jeglicher Hoffnung auf ein wenig verbleibende Utopie im Jahre 1965 im wichtigen Gedicht mit dem Titel «Geschichte» unmißverständlich, und auch als Leiden am deutsch-deutschen Zwiespalt anklingen:

6.

Sie ist über den Völkern.

An einem Faden.

Ein damokleischer Schatten: Deutschland

Unaufhörliche Wolke zwiefach zwieträchtiger Form.

Dabei wir dabei

Ins Universalische zu wachsen und an einem Kabel

In den Kosmos zu hängen zu schaukeln und frei

Uns zu fühlen vor allem von Gravitation von Atemnot von

Gedanken: einschneidenden ätzenden verletzenden Wie

Die dort unten die kleine bläuliche Kugel zerfurchten

[...]

7.

Geschichte sage ich und weiter noch: Wenig bleibt.

Glücklich wer am Ende mit leeren Händen dasteht

Denn aufrecht und unverstümmelt dasein ist alles.

Mehr ist nicht zu gewinnen ${ }^{10}$.

Auch im Roman gab es erste, wichtige Signale zu einem Umschwung, der die DDRLiteratur westlichen Literaturströmungen der Zeit wieder näher brachte: in zwei Romanen der Wiederentdeckung des Individuums und seines Glücksstrebens, Günter Kunerts erst 1976 in der DDR gedrucktem, bitter-ironischem Schelmenroman von 1967 über die Trümmerzeit und das verdrängte Holocaust-Thema mit dem Titel Im Namen der Hüte, sowie Christa Wolfs Nachdenken über Christa T. von 1968. Christa Wolf (geb. 1929) hatte um die Entstehungszeit des Romans einen Briefwechsel mit der jüngeren Schriftstellerin Gerti Tetzner (geb. 1936), die ihr auch ein Tagebuch schickte. Christa T. trägt möglicherweise Züge der Autorin, im Titel wären dann beide schreibenden Frauen enthalten. Dasselbe gilt für den Titel von Gerti Tetzners Karen W. (1974), ebenfalls ein früher, systemkritischer Roman mit einem frauenspezifischen Thema. Christa T. wächst in einem Dorf jenseits der Oder auf, ist nach dem Krieg für kurze Zeit Lehrerin, studiert dann Germanistik, heiratet einen Tierarzt, hat mit ihm Kinder, baut mit ihm ein Haus auf dem Lande, stirbt schließlich an Leukämie. Christa Wolf geht als Ich-Erzählerin anhand von Tagebüchern und persönlichen Aufzeichnungen der Freundin erstmals den Chancen der Selbstverwirklichung ihrer Protagonistin als Frau 
im sozialistischen Staat nach. Formelhaft gesprochen, treten hier erstmals «private» Fragen nach dem Glück in den Vordergrund eines DDR-Romans über eine Frühverstorbene, tritt Freud und ein neues Interesse an Natur und Romantik an die Stelle von Marx.

Der Blick, mit dem sie sich vom Auto aus nach den Kindern umsah, soll ein Abschiedsblick gewesen sein. Es wiederholt sich, was sich nicht wiederholen darf. Wiederholen, wieder zurückholen... Die Worte haben alle einen doppelten Sinn, einen aus dieser, den anderen aus jener Welt. Sie ist stiller und fragt weniger [...] Sie liest gierig. Sie nimmt die Gewohnheit wieder auf, Sätze, Zeilen zu notieren. Als letztes steht in ihrem Notizbuch ein Gedicht :

Wozu so teuflisch sich zerquälen?

Nie mehr gescheh, was da geschah:

Das Nahsein der sich fremden Seelen,

das Fremdsein derer, die sich nah...

[...] Es beginnt, was sie so schmerzhaft vermiß hatte:

daß wir uns selber sehen; [...] $]^{11}$.

Begnügt sich Christa Wolf hier nicht mit Anspielungen und indirekter Kritik an der DDR-Gesellschaft, etwa der Lehrerin T. gegenüber einem ehemaligen Schüler, jetzt Medizinstudent, der sich als Arzt mit «realer Existenz» und «Anpassung» des Menschen begnügen will, obwohl er sich von Christa T.s «phantastischer Existenz» einst faszinieren ließ, gibt sie mit Kindheitsmuster (1976) der mangelnden bisherigen Faschismusaufarbeitung in der DDR und anderen Reizthemen breiten thematischen Raum. In jenem Jahr war das Tauwetter seit Ulbrichts Wachablösung 1971 durch Honecker und der Scheinliberalität des 8. SED-Parteitags mit dem Tenor «tabufreien Schreibens», allerdings auf der Basis eines «festen sozialistischen Standpunkts», mit einem Eklat $\mathrm{zu}$ Ende gegangen. Wolf Biermann hatte in einem breit übertragenen November-Konzert in Köln die Tabus durch angeblichen Antikommunismus und Verletzung der «Treuepflicht» wieder sichtbar gemacht und wurde kurzerhand seines Visums beraubt. In den nächsten Tagen gab es eine Lawine von Solidaritätsunterschriften unter eine Petition zu seiner Rückeinbürgerung, vergebens. Jurek Becker, Volker Braun, Stephan Hermlin, Sarah Kirsch, Günter Kunert, Christa Wolf und ihr Mann Gerhard, kurz darauf Plenzdorf, Havemann, de Bruyn und die Filmschauspielerin Angelika Domröse gehörten zu den bekanntesten Unterzeichnern, viele wurden aus dem Schriftstellerverband ausgeschlossen und kaum mehr veröffentlicht, viele gingen über kurz oder lang in den Westen. Bier mann hatte unter anderem in Brechts und Heines Balladenton gesungen:

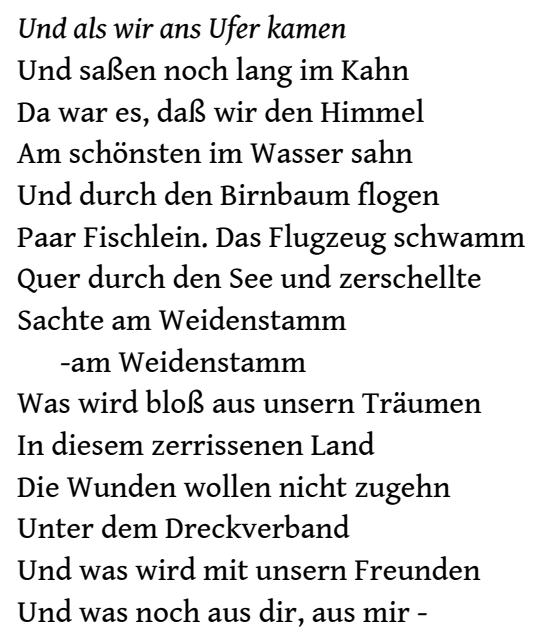


Ich möchte am liebsten weg sein

Und bleibe am liebsten hier

-am liebsten hier (LP CBS 13.11.1976)

19 Das heute prophetisch wirkende Gedicht einer zerrissenen, tagträumerischen Stimmung zwischen dem hochfliegenden Traum von Ufer und Ankunft und der Misere, das zerrissene Deutschland nicht hinter sich lassen zu können, keine Entlastung zu finden, darf nicht im bequem prowestlichen Sinne verstanden werden, das zeigen auch die gemischten Gefühle desselben Biermann angesichts der Wiedervereinigung. Er macht es sich und uns nicht leicht, darin dem Kunert der apokalyptischen Themen heute vergleichbar.

Eine Reihe von Prosatiteln zum deutsch-deutschen Thema in den 70er und 80er Jahren bietet sich in der DDR-Literatur an, während im deutschen Westen eigentlich nur Uwe Johnson mit den monumentalen Jahrestagen (1983 abgeschlossen), Peter Schneider mit Der Mauerspringer (1981), Thorsten Beckers satirische Erzählung Die Bürgschaft (1985) und Martin Walser mit der Novelle Dorle und Wolf (1987) in Frage kommen. Peter Schneider zeigt in seiner Collage von Mauerabenteuern die Schizophrenie und Absurdität der deutschen Teilung, aber auch die beharrenden, schwer zu überwindenden Trennungskräfte von ideologischen Administrationen, Selbstschußanlagen an der Mauer und preußisch verständnislosen DDR-Grenzbeamten sowie die Mauer im Kopf mancher Bundesdeutscher.

Thorsten Becker parodiert mit dem Thema der Schiller-Ballade nicht nur den unter Normalumständen zum fatalen Ausgang führenden Tausch eines West-Berliners in der DDR gegen einen von dort nach Wien gegangenen Freund vom Theater, also die moderne Variante der Ballade, sondern auch den «Erbe»-Gedanken der Becher-Jahre in der frühen DDR, darin Plenzdorf spiegelnd. Indem der Ich-Erzähler die Rückkehr des Freunds mit dem sprechenden Namen Schlitzer nach einer abenteuerlichen Schlußpointe samt gewaltsamem Ausweisraub in einer ostdeutschen Autobahnraststätte und drohender Verhaftung durch die Stasi noch zum Happy End wendet, macht er - ganz anders als es später Monika Maron in einer motivverwandten Episode ihres Romans Stille Zeile Sechs (1991) mit weit ernsteren Haftkonsequenzen für den Betroffenen erzählt - die bundesdeutsche Verharmlosung der Mauerzustände als Gewöhnung an die Teilung sichtbar. Reich-Ranickis Kommentar zur Becker-Satire markiert denn auch, vier Jahre vor dem Umbruch, die Undenkbarkeit jener späteren Wende von 1989: «Das geteilte Deutschland - das ist für Thorsten Becker schwerlich eine nationale Katastrophe, eher schon eine Selbstverständlichkeit. [...] Es ist keine Elegie, viel eher schon eine Parodie» ${ }^{12}$.

Martin Walser, der auch in seinen öffentlichen Kommentaren zur Wiedervereinigung, ähnlich wie Günter Kunert, als einer der wenigen deutschen Schriftsteller die neue Entwicklung als eine historische Chance begrüßte, beschreibt mit seinem Spionagethema die historisch überholte, kulturell unhaltbare Trennungssituation und das Leiden daran. Der spionierende Perfektionist Wolf aus dem Osten erhält von einem Bundesgericht am Ende fünf Jahre Haft, die beiden aus dem Westen kommenden Partnerinnen, die zuerst ahnungslose Ehefrau Dorle und die Sekretärin Sylvia, kommen mit Bewährung davon. Im Grunde geht es um das psychologische, eher «private» Leiden an einer im Liebesdreieck gespiegelten Gespaltenheit Wolfs, der seine schwäbische Frau Dorle am Ende mehr den je liebt und ersehnt, obwohl er sie mit 
Sylvia für seine Ostspionage betrog. Die Menschen der Bundesrepublik erscheinen Wolf als halbiert:

Wolf hatte im Westen entdeckt, wie sehr hier der Osten verlorengegangen war. Er hatte die zunehmende Kälte gegenüber allem Gemeinsamen erlebt und das grelle Unverständnis, die auftrumpfende Umempfind-lichkeit und Überheblichkeit gegenüber dem, was in der DDR tatsächlich geschah. Die Teile dröhnten vor Unverständnis füreinander. [...] Und dem wollte Wolf steuern, auf einem prekären Gebiet, dem der Rüstung nämlich. [...] Alles Verneinende fand er schwach. Alles was unfähig war, sich der ganzen Geschichte zu verbinden, kam ihm leblos vor» ${ }^{13}$.

Als Wolf seine eigene schizophrene Lage aufgeben will und aus der Spionage aussteigen, die ihm wie ein Stück Völkerverständigung erschien, ist es zu spät. Die Novelle endet mit Wolfs Unverständnis gegenüber dem bundesdeutschen Richter, der so denkt, als gäbe es kein geteiltes Land, und mit einem Stück Hoffnung, seiner Liebe zu Dorle, an die er aus dem Zuchthaus schreibt und an die er sich klammert.

Einige Titel ragen aus der DDR-Erzählprosa seit 1975 heraus, weil sie ästhetisch innovativ den sozialistischen Realismus links liegen lassen und zugleich unverblümt das eigene System sozio-politisch kritisieren. Es handelt sich um Texte von Volker Braun, Christoph Hein und Monika Maron.

Volker Braun gelingt in Unvollendete Geschichte (1975) eine Novelle im Kleist- und Büchnerton; die einmontierten Schlagworte und politischen Phrasen verbürgen, im Großdruck herausgehoben, authentisches Zeitrequisit im Gefolge Döblins, der lange aus den DDR-Bibliotheken verbannt blieb. Entstanden ist eine spätmoderne Collage um das Romeo-und-Julia-Thema in der DDR-Provinz. In Christoph Heins bekannter Novelle Drachenblut (1982), die ästhetisch mutig mit einer Traumsequenz einsetzt, die den Leser zur Deutung zwingt, ist der Erzählstandort die Innensicht einer scheinbar gegen alle Gefühle immun gewordenen Ostberliner Ärztin. In Der Tangospieler (1989) zeichnet Hein das Porträt eines fast zwei Jahre für seine politischen Überzeugungen inhaftierten Historikers, ein noch im Jahr darauf durch die DEFA verfilmter Roman, der die Wende antizipiert. Auch Monika Marons in der DDR nicht mehr erschienener Collage-Roman Die Überläuferin (1986) zeigt die Fluchtphantasien einer Historikerin, die bis nach New York führen. Die 1941 in Berlin geborene Monika Maron übersiedelte 1988 mit Mann und Sohn nach Hamburg, zunächst mit einem Drei-Jahresvisum. Keiner ihrer Romane, am bekanntesten Flugasche (1981), konnte in der DDR erscheinen.

Doch zunächst zu Volker Brauns Unvollendeter Geschichte (1975):

Am Tag vor dem Heiligabend eröffnet der Ratsvorsitzende des Kreises K., wie seine Frau zum politischen Establishment der DDR gehörend, der achtzehnjährigen Tochter Karin, sie müsse sich von ihrem Freund Frank trennen. Später stellt sich erst heraus, daß Frank, Einzelgänger, Rowdy und alles andere als ein politisch konformer Typ, im Verdacht steht, zu Verwandten im Westen zu fliehen. Karin ist Volontärin an einer Bezirkszeitung. Für sie steht zunächst zuviel auf dem Spiel und sie gehorcht, zieht sogar fort in die nahe Bezirksstadt, ohne Frank Genaueres zu erklären. Sie glaubt zunächst noch an den Sozialismus. Frank nimmt eine Überdosis Schlaftabletten weil er außer ihrer Liebe nichts hat. Als sie zufällig nach ihm sieht und, seinen gefährdeten Zustand unterschätzend, wieder fortgeht, dreht er das Gas auf und erleidet ein Koma, bevor er gefunden wird.

Karin entdeckt nun mit ihren Schuldgefühlen ihre Liebe und stellt sich gegen das unmenschliche System. Sie ist zudem schwanger, verliert aber trotz ihrer anfänglichen Anpassungswilligkeit die Stelle in der Zeitung; der stark-schwache Vater kann in der Redaktion nichts bewirken; der Fall soll niedergeschlagen werden, nur nicht mehr davon reden. Karin soll «zur Bewährung in die Produktion» 
und versteht die Welt nicht mehr, kann nun wegen ihrer Schwangerschaft und Treue zu Frank auch keine Stelle mehr finden, bis nach Monaten ein Bekannter verspricht, sich einzusetzen. "Sie ging zum Rat der Stadt. Ein Genosse hörte sie an. Er erkundigte sich bei der Redaktion. Er sagte: So gehts nicht. Sie können nicht wochenlang nach Arbeit suchen. Ich helfe Ihnen, ich werde Sie irgendwo unterbringen». Frank, kann nach vier Monaten wieder das Bett verlassen: «Sie wollte Frank an sich drücken, aber er taumelte, er war noch schwach. Sie hatte Angst, daß er gleich umfällt. Sie mußte ihn halten. Sie standen umschlungen auf der Straße. Die Leute, die vorüberkamen, blieben stehn. [...] Die Beiden hielten sich bleich aneinander fest. Sie starrten sich an. [...] Sie ließen sich nicht los.

Hier begannen, während die eine nicht zuende war, andere Geschichten» ${ }^{14}$.

In der bundesdeutschen Kritik wurde die lakonische und doch im Büchner-, Kleist-, und Werther-Ton unauffällig moderne Novelle zwischen zwei Liebenden und ihren Familien, die für und gegen das System stehen, als eines der wichtigsten DDR-Bücher überhaupt behandelt, «das erregendste und ernsteste Stück Prosa» seit Christa Wolfs Nachdenken über Christa T., wie Die Zeit urteilte. In der DDR wurde das nur in kleiner Auflage in der mutigen Zeitschrift Sinn und Form erschienene Werk kaum rezipiert. Die chronologisch vorgetragene Handlung, durchbrochen von kurzen Rückblenden, erhält ihr authentisches Gewicht durch den knappen, dokumentarischnüchternen Stil, der dennoch poetisch vibrierende Momente des Lyrikers Braun birgt. Den Leser sollen die in Großbuchstaben gedruckten Stereotypen und politischen Phrasen wachrütteln, "private», und damit der Selbstverwirklichung dienende Urteile und Gefühle erscheinen durch Kursivdruck hervorgehoben. Das Ganze vermag aus der Sicht Karins, die nie die Hintergründe genau erfährt, den Leser zu motivieren, da aus einem nicht überprüften politischen Verdacht unmenschliches Handeln und dadurch menschliches Leiden entsteht. Schuld an allem soll angeblich der «Klassenfeind» sein. Entstanden ist eine der gültigsten Anklagen gegen den Stasistaat des «Sozialismus von oben», umso überzeugender, als der Autor die großen Hoffnungen des Beginns mit seiner Lyrik begleitete. Karin, die sich wandelnde Heldin, begreift ihre existentielle Ausgesetztheit, als sie schwanger wird:

Sie hatte sich BENOMMEN, daß ihr die Prädikate «brav» und «artig» zugesprochen wurden, sie war nie AUS DER REIHE GETANZT, lieber hatte sie die Reihe angeführt: im Gruppenrat der Klasse, im Freundschaftsrat der Schule. Siebenmal an sieben verschiedenen Schulen, während der Vater in den Funktionen stieg, und es war für sie das Beste: sie hatte sich durchbeißen müssen. Das sah sie ja!

Jetzt sah sie nichts mehr. Sie wurde schwanger. In den ersten Tagen vergaß sies immer wieder, oder sie dachte an sich wie an einen andern Menschen: die kriegt ein Kind, stell dir das vor! - aber erschrak dann, daß sies selber war, und das in ihr vorging und wachsen würde, sie hatte nichts damit zu tun. Dann dachte sie, sie habe nichts dagegen. Für die Gedanken konnte man, der Körper ging keinen an. [...] Für all die Theorien war er nicht zuhaus. [...] Nur manchmal, wenn sie ganz ruhig war und zu sich kam, drehte sich alles heraus an ihr, sie war das Fleisch und die Haut, und das Haar und empfand sich mit allen Fasern, lag so da. Erinnerte sich entfernt an irgenwelche Gedanken, an denen sonst alles hing, die schwammen so weg, und lächelte weit weg von jedem Grund ${ }^{15}$.

Auch Christoph Heins Drachenblut verwendet bis auf den düsteren Eingangstraum als Inneren Monolog eher eine unauffällig moderne, chronologische Erzählweise, wenige Rückblenden im Leben einer nüchtern denkenden Ärztin im mittleren Alter, die nach dem gewaltsamen Tod des Freundes Henry durch eine Zufallschlägerei jugendlicher Rowdys gegen alle weiteren Verletzungen gewappnet scheint. Jedoch ist ihre wachsende Entfremdung von den Menschen subtil und mit kritischer Einfühlung 
gezeichnet. Der Autor läßt in einer wüsten Szene auf einer Landfahrt erkennen, daß sie Henry weit mehr liebte, als sie sich selbst gesteht, und daß ihre Single-Fassade alte Wunden nur notdürftig deckt: die Erinnerung an eine frühere beste Freundin Katharina, die, christlich erzogen und mit Westverbindungen, von der Lehrerin systematisch aus der Klasse geekelt wurde, als sie nicht in den sozialistischen Jugendverband wollte. Die Ärztin und Ich-Erzählerin half dabei mit. Am Ende bricht die Fassade auf und gibt systemkritisch zu denken:

Es läuft alles in seiner gewohnten Ordnung, alles normal. Kein Anlaß für einen Schrei. [...] Ich habe in Drachenblut gebadet und kein Lindenblatt ließ mich irgendwo schutzlos. Aus dieser Haut komme ich nicht mehr heraus. In meiner unverletzbaren Hülle werde ich krepieren an Sehnsucht nach Katharina ${ }^{16}$.

Rettung in dieser Fassade der Gleichgültigkeit käme nur noch von neuer Verletzlichkeit, einem Lindenblatt. Während das Berufs-und Singlethema in einem lieblosen Berliner Appartement sicher in vielem ebensogut im Westen spielen könnte, läßt Hein in seiner Novelle doch mit dem Katharina-Schulerlebnis keinen Zweifel an seiner DDR-Kritik, die er in Horns Ende (1985) verschärft. Es geht um den von fünf Menschen erinnerten Selbstmord eines unangepaßten, jungen Museumsdirektors in den 50er Jahren, der Opfer einer Denunziation wird. In dem späten Stück Die Tafelrunde (1989), kurz vor dem 9. November aufgeführt, zeigt Hein das Ende aller alten Utopien der ersten Stunde.

In Christoph Heins Der Tangospieler (1989), noch vor der Maueröffnung veröffentlicht, ist es wiederum ein Historiker, Dallow, der seine erschreckend ungerechte, zweijährige Haftstrafe für das Einspringen als Tangospieler bei einem Studentenkabarett und einem Ulbricht verspottenden Lied danach zunächst durch totale Weigerung quittiert. Die im Laufe des erzählten Jahres 1968 in der Tschechoslowakei nach dem Prager Frühling erfolgte Repression mit Hilfe von Warschauer-Pakt-, darunter DDR-Panzern lassen seine Bemühungen, trotz Stasi-Überwachung lieber als Kraftfahrer und Kellner zu arbeiten, als parteifromm ins Leipziger historische Institut zurückzukehren, zu einem überraschenden und ambivalenten Ende kommen: Der statt seiner im Institut aufgestiegene Dozent gerät in politische Ungnade, weil er nicht an die DDR-Teilnahme an der Prager Unterdrückung glauben wollte und sich nicht rasch genug anpassen kann. Der aller sozialistischen Illusionen beraubte Dallow übernimmt seine Position. Im Laufe des Jahres hat er entdecken müssen, daß sich viele Menschen bei der Ausübung ihres Berufs angesichts möglicher Fehler immer «mit einem Bein im Zuchthaus» fühlen: entstanden ist das Porträt eines alle umfassenden DDR-Gefängnisses.

Schließlich findet die durch eine jüdische Mutter im nicht geheuren Berlin des Dritten Reichs sensibel gewordene Monika Maron - die zweite Ehe der Mutter mit dem späteren DDR-Innenminister Karl Maron schärft eher den kritischen Blick der Autorin für die Macht - mit dem Thema der Überläuferin (1986) hellseherisch die Konstellationen für Probleme der DDR-Schlußphase. Eine in die psychische Krankheit flüchtende Historikerin Rosalind sieht sich mit zwei engen Freudinnen, vor allem der frauenbewegten Martha auf vielen Stationen phantastischer Flucht. Der langjährige Freund Bruno, zynisch und abgeklärt, kann ihr nicht helfen, das New York der Freundin Martha ist für sie keine Alternative. Am Ende kehrt sie wieder zu neuer IchStärke zurück, nahe einem neuen Naturbewußtsein, fort vom System. Dies erscheint in einer gigantischen Collage korrupter und skurriler Funktionärsfiguren, Schriftstellerorganisatoren, geklönter Spione des Systems, faschistischer Obdachloser und stereotyper Figuren wie der «Frau mit der eigenen Meinung». Erzählbruchstücke 
werden szenisch, wie auf der Bühne stilisiert, wiedergegeben, die Journalistin und Theaterassistentin Maron findet hier satirische und bunte Bilder in der Nähe von Irmtraud Morgner. Die am Ende nicht mehr gelähmte Heldin findet sich im Ostberliner Zimmer wie in einem Gefängnis wieder, alles scheint enger geworden:

Ein dünnfädiger Regen belegte den trüben Novembermorgen mit eisigem Glanz. Es war, es ist, es wird sein; wie Schlangen verknäulten sich die Zeiten zu diesem Augenblick, in dem Rosalind sich unversehens wiederfand. [...] Als würde sie vom falschen Ende durch ein Fernglas sehen, schrumpfte alles, was sie umgab, auf ein fernes unwirkliches Maß [...] Von draußen hörte sie das Rauschen des anschwellenden Regens, den der Wind durch die Straßen trieb. [...] Den Mund weit öffnen und das Wasser in mich hineinlaufen lassen, naß werden, dachte sie, vom Regen naß werden, ja das wäre schön ${ }^{17}$.

Auch hier also ein Bild der geschichtslosen Natur als Gegenbild zur wie unwirklich schrumpfenden DDR-Realität, Existenz als Wiedererlernen der einfachen Grundbedürfnisse jenseits der Politik. Dagegen steht die Weiträumigkeit phantastischer Reisen, Korrelat der ersehnten Freiheit. Eine aufchlußreiche Schlußpointe. In einem Interview 1988 beklagte die Autorin, nach Verlassen der DDR, die dortige Lustlosigkeit, das mürrische Anstehen nach allem, den geringen geistigen Pluralismus für Autoren:

Von den großen Ideen ist viel mehr als rechthaberisches Preußentum nicht übriggeblieben - und selbst das funktioniert nicht.

«Ein bißchen weniger Zensur» gebe es eben nicht, «es ist alles zu wenig und zu spät» ${ }^{18}$.

Monika Maron sollte recht behalten. Ihr Roman Stille Zeile Sechs (1991) ist vielleicht der bislang wichtigste und moralisch eindrucksvollste Beitrag zur Abrechnung mit der politischen und soziokulturellen DDR-Vergangenheit, erlebt von einer immer schon systemkritischen Autorin, ein Blick zurück im kalten Zorn, der durch seinen über große Strecken eher nüchternen Realismus auch einem großen Leserkreis zugänglich bleibt. Der Roman wurde, wie Fritz Rudolf Fries in seiner Rezension berichtet, noch in der DDR begonnen. Fries, dessen ästhetisch innovativer und alles andere als systemkonformer Roman von 1966, Der Weg nach Oobliadooh, erst 1989 im Aufbau-Verlag erscheinen konnte, vermag in seiner Rezeption des Maron-Romans die aufklärenden Qualitäten dieser politischen Generalabrechnung aus der Sicht des Mitbetroffenen zu spiegeln:

Das ist der Roman, den zu schreiben uns zur Aufgabe gemacht wird vom mahnenden deutschen Feuilleton. Der Roman, der mit uns abrechnet und erzählt, wie es war. [...] Die Rosalind Polkowski beider Romane verweigert sich den Leistungsnormen der Gesellschaft, der sie am historischen Institut [...] genügen soll. Sie will frei und wenn es sein muß verfemt sein wie eine Katze.

Da trifft sie in einem Café, wie man es in den späten achtziger Jahren Unter den Linden finden konnte, den Rentner Beerenbaum, der genußvoll seine Apfeltorte verzehrt. Seine rechte Hand ist gelähmt, also schreibunfähig. Aber auch er möchte der Nachwelt mitteilen, warum er gelebt hat. Er gewinnt Rosa, die so tut, als sei er ein alter Mann wie viele andere, als Schreibkraft. Beerenbaum diktiert ihr sein unreflek-tiertes, nicht in Frage zu stellendes Leben in seiner Wohnung in Pankow, im «Städtchen», wo einst alle Bonzen wohnten, bevor sie sich in Wandlitz der Öffentlichkeit entzogen. [...]

Zugegeben, Monika Marons Geschichte ist von gestern: ihr leises Moralisieren scheint mit dem Fall der Mauer anachronistisch geworden zu sein, im Zwiegespräch von Rosa und Herbert. Der eine, der alternde, an der Schwelle des Todes stehende Hochschulbeamte Herbert Beerenbaum, ist autoritär. Er hat auch jetzt noch immer recht, wie seine zur Zeit der Handlung noch regierende Partei. Die zweiundvierzigjährige Historikerin Rosalind Polkowski hat schlechte Erinnerungen, die mit der Unaufmerksamkeit ihres Vaters, eines Schuldirektors, beginnen. [...] 
Nun ist Herbert Beerenbaum kein Mörder. Der Arbeitersohn aus dem Ruhrgebiet hat als Emigrant aus der Sowjetunion, als überlebender Gast des «Hotel Lux» eine makellose Kaderakte; mehr noch, er ist ein Mensch, ein glücklicher Heimkehrer, der seine Grete am Leben trifft und in Liebe zu ihr die neue Gesellschaft aufbaut. Schuldig geworden ist er durch zuviel Macht, die ihm durch zuviel Bürokratie in die Hände gegeben wird, oder war es umgekehrt. Für drei Jahre hat er den Studenten Baron, in Rosalinds Lieblingskneipe der Graf genannt, eines Manuskripts wegen ins Gefängnis gebracht. [...]

Als einmal der Schriftsteller Victor Sensmann - den man wie Beerenbaum zu kennen glaubt, ohne ihn im wirklichen Leben benennen zu können - den Alten besucht und beide Papier reden und die Mauer bejahen, kommt es zum Aufstand der fügsamen Schreiberin. Als spräche jede Generation eine andere Sprache, wobei der pädagogische Ansatz der Erwachsenen dem Kind Rosalind schon immer die Lüge als Wahrheit angeboten hat. Da bleibt nur ein Berührungspunkt, der Rosas Zorn neutralisiert. Was sie in der Nazizeit gewesen wäre, fragt Beerenbaum: «Vielleicht wäre ich Kommunist geworden», sagt Rosa. [...] Die Klugheit der Autorin ist allemal größer als die Verachtung und der Zorn Rosas auf Beerenbaum, diesen Inbegriff eines Chefs, der seine Rechte nicht aufgeben will. [...] Der Verstorbene hat es so gewollt, daß seine Memoiren in ihren Besitz übergehen. [...] Wie die Autorin wird zumindestens diese Generation von diesen Papieren nicht loskommen ${ }^{19}$.

Auch wenn Monika Maron die Honeckers und Hermann Kants in ihrer Unbelehrbarkeit ebenso aufdeckt wie moralisch differenzierend abwägt, ihre ätzende Präzision im Bloßstellen der Vätergeneration samt deren Anpasserei und inhumanen Sprachregelung ist gerade im neuerdings durchgehaltenen Realismus überzeugend. Rosalind wünscht sich den Tod des alten, auch ihre Frauenwürde nicht achtenden Funktionärs und führt ihn durch ihre Anklagen mit herbei. Der «Klasseninstikt» ihres Vaters, der Kafka «dekadent» und «schädlich» nennt, das Gerede Beerenbaums vom «sozialistischen Schutzwall», "guten Genossen» und der «richtigen Perpektive» - all jene Phrasen des abgelebten Systems erscheinen im Licht der subtilen Psychogramme und inhumanen Handlungsweisen im Alltag zweier DDR-Gründerväter doppelt unaufrichtig.

Die im März 1991 erschienene, lange Erzählung von Friedrich Christian Delius, Die Birnen von Ribbeck, versucht aus West-Berliner Sicht Fontanes Ballade zu einer Geschichtsrekapitulation von hundert Jahren Havelland seit Erscheinen des Gedichts zu formen. Entstanden ist eine Sympathieerklärung des Autors für die geschichtsgeprüfte Region im Bewußtseinsstroms eines Havelbauern. Die Westberliner erobern Ribbeck mit ihren teuren Schlitten und Videokameras, nehmen den Dorfbewohnern mit den «mächtigen Markstücken» die Ruhe und den Rest Identität und haben für den roten Faden der Dorfgeschichte, auch die dortige stille Liebe zu Fontane, wenig Geduld. Der originelle, streckenweise poetisch verdichtete Versuch, zentriert um eine symbolisch vielschichtig und relevant gemachte Ballade des «Erbe»-Dichters Fontane, ist nicht frei von Klischees typischer DDR-Vorbehalte gegenüber bundesbürgerlicher Naivität. Die zustimmende Gewöhnung ans westliche Konsumverhalten im allabendlichen DDRFernsehen lange vor der Maueröffnung läßt der einstige Wagenbach- und RotbuchLektor Delius für sein verdichtetes Genrebild beharrlich außer acht. Sein Versuch steht für das Bedauern manches Westautors, daß die Maueröffnung zu schnell kam, am eigensinnigsten von Günter Grass vorgebracht in Deutscher Lastenausgleich. Wider das dumpfe Einheitsgebot. Reden und Gespräche (1990) und in Ein Schnäppchen namens DDR (1990). 
Zwei Aspekte dieses stark auswählenden Überblicks der letzten zwanzig Jahre kultureller Wiederannäherung können der Zusammenfassung dienen: Günter de Bruyns These der nie ganz geteilten Kulturnation findet Bestätigung gerade in der auffälligen Wahl von Historiker-Protagonisten bei Hein und Maron. Die eine Geschichtsschreibung hüben wie drüben ergänzend, korrigierend, lebendig und subjektiv begleitende Reihe literarischer Texte hat dem Historismus immer die offen haltende, leserrelevante Ich-Perspektive als ein Stück Selbstfindung und Mentalitätsgeschichte entgegengesetzt. Historiker als häufige Hauptfiguren von DDRRomanen des letzten Jahrzehnts betonen diesen Zusammenhang und die besondere Sensibilität der Helden gegenüber einer gemeinsamen, wenn auch nur latent vorhandenen, Kulturnation.

37 Als den Mauerschüssen vergleichbares Skandalon im intellektuellen Bereich muß die Zensur und Stasi-Überwachung der Autoren bis zu deren Verstummen oder Ausbürgerung gelten. $\mathrm{Zu}$ diesem traurigen Kapitel gibt es nun am Beispiel einzelner Autoren wie Kunert, Kunze oder Jurek Becker erste Versuche der Aufarbeitung ${ }^{20}$. Im Roman jedoch konnte, nach immer noch verhüllten Hinweisen Monika Marons und Christoph Heins im Werk bis 1989, erst Stille Zeile Sechs (1991) mit einer so eklatant moralischen und anrührenden Szene aufwarten, wie die Reaktion der Historikerin Rosalind Polkowski auf das «Antifaschisten-Schutzwall»-Gerede zweier Altfunktionäre unter den Intellektuellen:

Allein die Zumutung, das Wort hinzuschreiben, als wäre es ein Wort wie Blume, Hund und Mauer, empörte mich. Ich notierte: B: Zeit nach Bau des Anti-faschuwa war aufregend. [...] Damals, sagte Beerenbaum, vor dem historischen August 61, habe er, wenn er morgens beim Betreten der Universität die Linden hinunterblickte, oft die Vision gehabt, Ströme des Lebenssaftes der jungen Republik, rot und pulsierend, durch das Brandenburger Tor geradewegs in den gierigen Körper des Feindes fließen zu sehen. [...] Sensmann durfte den offenen Widerspruch nicht wagen und ich sollte ihm helfen.

Da haben Sie das Blut lieber selbst zum Fließen gebracht und eine Mauer gebaut, an der Sie den Leuten die nötigen Öffnungen in die Körper schießen konnten, sagte ich.

Zwei oder drei Sekunden lang war es so still, als hielten wir alle drei den Atem an ${ }^{21}$.

Als Stefan Heym 1989 sagte, «Es wird keine DDR mehr geben. Sie wird nichts sein als eine Fußnote in der Weltgeschichte», konnte er die von großem Leserinteresse im Westen, nicht nur in der Bundesrepublik, begleitete DDR-Literatur nicht gemeint haben. Zu einer Neubewertung ist es noch sehr früh. Die stärksten Kontraste bestanden in den Jahren 1951 bis 1971, als der sozialistische Realismus Doktrin war. Aber bereits sehr früh, schon seit 1965, vollzog sich eine neuerliche Annäherung an Themen und Formen westlicher Spätmoderne. Immer blieb dabei aber ein integraler Bestandteil die kulturelle und soziopolitische Wirklichkeit der DDR, die die Literatur dort unverwechselbar und bis zum Ende färbte. Formen der Postmoderne allerdings, wie sie bei uns Peter Handke, Botho Stauß und Hanns-Josef Ortheil seit Mitte der 70er Jahre versuchten, erscheinen auch im Rückblick in der DDR-Literatur dünn gesät. Bei der Prenzlauer Berg Generation der nach 1950 Geborenen könnte man auf Katja LangeMüllers Collage-Text Kaspar Mauser. Die Feigheit vorm Freund (1988) hinweisen, oder auf Angela Krauß' Sprachskepsis im Prosaband Das Vergnügen (1989), wo gerade die hohlen Phrasen einer Betriebsfeier der «Brikettfabrik Rosdorf» in ironischer Anspielung auf Bitterfeld den Beteiligten im Munde stecken bleiben ${ }^{22}$. Die drei DDR-AutorenGenerationen haben einen langen Weg zur Spätmoderne zurückgelegt, einen auch für 
uns lehrreichen und unverwechselbar eigenen Weg, der die deutsche Literatur nachhaltig bereichert.

\section{NOTES}

1. - Jens Reich: «Altweibersommer. Erlebnissplitter und Gedankenfetzen, Deutschland im Jahre 93», In Lettre International 5 (1993), H. 22, S. 6.

2. - Ralf Schnell: Geschichte der deutschsprachigen Literatur seit 1945, Stuttgart, Weimar 1993, S. 226

f.

3. - Wolfgang Emmerich: «Zwischenbilanz 1992. Einheit und Vielfalt der deutschen Literatur», in: Beutin u.a.: Deutsche Literaturgeschichte, 4. Aufl. 1993, S. 606-612.

4. - Günter de Bruyn: «So viele Länder, Ströme, Sitten. Gedanken über die deutsche Kulturnation», in: Jubelschreie, Trauergesänge, Frankfurt a.M. 1991, S. 21, 25.

5. - Günter de Bruyn: «Deutsche Befindlichkeiten», in: Jubelschreie, S. 37.

6. - Günter Kunert: Fremd daheim. Gedichte, München: Hanser, 1990, S. 117.

7. - Vgl. Günter Kunert: Vor der Sintflut. Das Gedicht als Arche Noah. Frankfurter Vorlesungen, München: Hanser, 1985, S. 53. Der Atlantis-Bezug zu Berlin wird dort deutlich: «Dieses Vineta unserer Tage, untergegangen durch Krieg, Teilung, Wiederaufbau, Sanierung, ist für mich, der dort geboren wurde, mehr und mehr versunken».

8. - Ebd., S. 46.

9. - Ebd., S. $47 \mathrm{f}$.

10. - Günter Kunert: Verkündigung des Wetters. Gedichte, München: Hanser, 1966, S. 25-28.

11. - Christa Wolf: Nachdenken über Christa T. [1968], Neuwied u. Berlin: Luchterhand, 1971 (SL 31), S. 231.

12. - Zitiert nach Thorsten Becker: Die Bürgschaft. Erzählung, Köln: Kiepenheuer, 1987 (KiWi 135), S. 166.

13. - Martin Walser: Dorle und Wolf. Eine Novelle, Fa. M.: Suhrkamp, 1987, S. 147-149.

14. - Volker Braun, Unvollendete Geschichte (1977), Fa. M.: Suhrkamp, 1989: (st 1660), S. 95-97.

15. - Ebd., S. $30 \mathrm{f}$.

16. - Christoph Hein: Drachenblut (1982), Darmstadt u. Neuwied: Luchterhand, 1985 (SL 616), S. 172 .

17. - Monika Maron: Die überläuferin Roman (1986), S. 220 f.

18. - Volker Hage: «Alles zu wenig, alles zu spät. Steht die Kulturpolitik der DDR vor einer Wende?», Die Zeit, 17.5.1988.

19. - Fritz Rudolf Fries: «Von Rosa und Herbert», Stuttgarter Zeitung, 29.2.1992.

20. - Vgl. etwa Manfred Durzak: «Die Widerstandskraft der Literatur. Zu der Kurzgeschichte Bericht des Zensors über die Begegnung mit einem gewissen G. von Günter Kunert», in: Günter Kunert. Beiträge zu seinem Werk, hg. M. Durzak, H. Steinecke. München: Hanser, 1992, S. 190 ff., mit acht Seiten Dokumentation zu Kunert, Kunze und der Zensur. - Erich Loest: Die Stasi war mein Eckermann oder: mein Leben mit der Wanze. Göttingen: Steidl, 1990. - Jurek Becker: Warnung vor dem Schriftsteller. Drei Vorlesungen in Frankfurt. Fa. M.: Suhr kamp 1990.

21. - Monika Maron: Stille Zeile Sechs. Fa. M.: Fischer, 1991, S. 107 f.

22. - Vgl. die Überblicksdarstellungen: Uwe Wittstock: Von der Stalinallee zum Prenzlauer Berg. Wege der DDR-Literatur 1949-1989, München: Piper 1989. - Wolfgang Emmerich: Kleine Literaturgeschichte 
der DDR. Erw. Ausg. Fa.M.: Luchterhand, 1990. - Volker Hage: Schriftproben. Zur deutschen Literatur der achtziger Jahre. Reinbek: Rowohlt, 1990. - Günter Rühle: «Das zerrissene Theater. 1991: Rückblick auf die Szene des jahrunderts», Neue Rundschau, 103 (1992), H.1, S. 154-169.

\section{RÉSUMÉS}

Die Untersuchung des Zeitromans, vorwiegend in der DDR, aber auch einiger Gedichte, zeigt seit 1965, intensiver seit 1971 (dem DDR-Machtwechsel von Ulbricht zu Honecker) eine ästhetische und kulturelle Wiederannäherung an die westdeutsche Spätmoderne in der Literatur. Die literaturkritische These läßt sich anhand der Gedichte und Romane belegen, aber wäre auch im Drama deutlich, daß der bürgerliche deutsche Westen und der sozialistische Osten nur in den «frühen» Jahren nach 1949 eine fundamental andere Literatur hervorbrachten, dann konvergiert seit 1965 inkurzen Texten, seit 1971 in der Romanprosa die Literatur zur westlichen, zunehmend psychologisch sensiblen Thematik, fort vom «Sozialismus von oben» und vom sozialistischen Realismus des «Bitterfelder Wegs». Eine kulturelle These läßt sich an den ausgewählten Texten ebenso erhärten: sie folgt Günter de Bruyns Konzept von der über lange Zeit - spätestens seit der Aufklärung - bestehenden gemeinsamen Kulturnation: die vierzig Jahre DDR konnten hier ebenfalls, deutlich seit 1971, keine dauerhafte Trennung in zwei kulturelle Deutschland bewirken und die Mauer hat hier zusätzlich Dissidenten gegen solche Unterdrückung unter den DDRAutoren hervorgebracht. Die hier untersuchten Autoren sind in der DDR Christa Wolf, Günter Kunert, Heiner Müller, Monika Maron, Christoph Hein und Volker Braun. Auf die Schriftsteller des Prenzlauer Bergs wird kurz hingewiesen, ebenso auf den erst seit 1989 mit einer systemkritischen Thematik hervortretenden Wolfgang Hilbig. Im Westen sind es vor allem Martin Walser, Thorsten Becker, F.C.Delius und Peter Schneider, die mit deutschdeutschen Themen die Wiederannäherng signalisieren.

L'étude du roman d'actualité, surtout en RDA, l'étude également de quelques poèmes montrent qu'on s'y est rapproché depuis 1965, et plus encore depuis 1971 (lorsque Honecker a succédé à Ulbricht), au plan esthétique et culturel, du modernisme littéraire tel qu'il s'est présenté ces temps derniers en Allemagne de l'Ouest. Du point de vue littéraire, on peut étayer cette thèse en se référant aux poèmes et aux romans. Elle est confirmée tout aussi nettement par le théâtre. Les littératures de l'Ouest bourgeois et de l'Est socialiste n'ont été fondamentalement différentes que durant leur "premiers temps » après 1949 ; en revanche, depuis 1965 pour ce qui est des textes courts, et depuis 1971 dans le roman, il se fait jour dans la littérature est-allemande une convergence avec la thématique occidentale et sa sensibilité psychologique, un éloignement du «socialisme imposé par ceux d'en haut» et du réalisme socialiste du «Bitterfelder Weg». De même, les textes sélectionnés pour cette enquête permettent de soutenir une thèse concernant le domaine culturel en général, qui est inspiré de celle qu'a formulée Günter de Bruyn: à savoir que l'Allemagne forme depuis longtemps une «Kulturnation», au moins depuis l'ère des Lumières ; les 40 années qu'a duré la RDA n'ont pas pu (et c'est encore devenu plus net à partir de 1971) instaurer de séparation culturelle véritable entre deux Allemagnes. Le Mur n'a fait que renforcer la résistance contre cette sorte d'oppression, parce qu'il a créé un phénomène de dissidence parmi les auteurs de la RDA. Les auteurs étudiés dans cet article sont pour la RDA: Christa Wolf, Günter Kunert, Heiner Müller, aron, Christoph Hein et Völker Braun. Il est question brièvement des écrivains du Prenzlauer Berg, ainsi que de Wolfgang Hilbig dont la critique du « système » n'a 
débuté qu'en 1989. A l'Ouest, ce sont surtout Martin Walser, Thorsten Becker, F.C. Delius et Peter Schneider qui ont marqué le rapprochement, en traitant des thématiques inter-allemandes. 